\title{
Light Guidance in Photonic Band Gap Guiding Dual Ring Light Cages implemented by Direct Laser Writing
}

\author{
Bumjoon Jang ${ }^{1}$, Julian Gargiulo ${ }^{2}$, Ron Fatobene Ando ${ }^{1}$, Alberto Lauri ${ }^{3}$, Stefan A. \\ MAIER $^{2,3}$, AND MARKUS A. SCHMIDT ${ }^{1,4},{ }^{*}$ \\ ${ }^{1}$ Leibniz Institute of Photonic Technology, Albert-Einstein-Str. 9, 07745 Jena, Germany \\ ${ }^{2}$ Chair in Hybrid Nanosystems, Nanoinstitute Munich, Königinstrasse 10, Ludwig-Maximilians-Universität Munich, 80539 Munich, Germany \\ ${ }^{3}$ Department of Physics, Imperial College London, London SW7 2AZ \\ ${ }^{4}$ Otto Schott Institute of Materials Research (OSIM), Friedrich Schiller University of Jena, Fraunhoferstr. 6, 07743 Jena, Germany \\ *Corresponding author: markus.schmidt@leibniz-ipht.de
}

Compiled July 14, 2019

Efficient waveguiding inside low refractive index media is of key importance for a great variety of applications that demand strong light/matter interaction on small geometric footprints. Here we demonstrate efficient light guidance in single-defect dual-ring light cages over millimeter distances that are integrated on silicon chips via direct laser writing. The cages consist of two rings of high aspect-ratio polymer strands (length $5 \mathrm{~mm}$, aspect ratio $>1000$ ) hexagonally arranged around a hollow core. Clear core mode formation via the photonic band gap effect is observed, with the experiments showing pronounced transmission bands with fringe and polarization contrasts of $>20 \mathrm{~dB}$ and $>15 \mathrm{~dB}$, respectively. Numerical simulations confirm our experiments and reveal the dual-ring arrangement to be the optimal geometry within the light cage concept. Particularly, the side-wise access to the core regions and the chip-integration makes the light cage concept attractive for a great number of fields such as bioanalytics or quantum technology. (๑ 2019 Optical Society of America

\section{http://dx.doi.org/10.1364/ao.XX.XXXXXX}

Strong light-matter interaction inside an optical waveguide is of essential importance for many fields and can be realized by guiding the light directly inside the medium of interest. Such direct guidance is particularly difficult to achieve in case of materials that have a refractive index (RI) that is so low that total internal reflection is suppressed. However, investigating light/matter interaction of such materials in a waveguide environment is relevant for a great number of applications such as bioanalytics [1], nonlinear light generation [2] and atomic spectroscopy [3].

Particularly within fiber optics, the mentioned applications have triggered substantial interest in developing novel concepts for hollow-core fibers in order to confine the electromagnetic energy close to $100 \%$ inside the low index core region via nanoor microstructured claddings. Beside photonic band gap (PBG) [4], Omniguide [5], metamaterial-based [6] or Kagome fibers
[7], fibers that guide via the anti-resonant effect have gained substantial interest due to a simplified fabrication scheme and ultralow loss [8]. All mentioned fibers include a cladding encapsulating the core region from the environment, thus allowing materials to be introduced only via the fiber ends, which can limit applications that demands to quickly exchange the core material (e.g., alkali gasses in low vapor pressure [9]).

Within planar waveguide technology the access of the spatial domain perpendicular to the substrate plane is considerably more challenging than for its in-plane counterparts, making the implementation of hollow core waveguides using planar technology difficult as out-of-plane confinement is hard to achieve. Here, the most widely used approach relies on a hollow core that is surrounding by a dielectric multilayer, leading to antiresonantreflecting-optical-waveguides (ARROWs) [10]. Even though being successfully employed in quantum technology [11] and bioanalytics [12], difficult fabrication, encapsulating geometry and modal incompatibility with fiber optics have prevented the widespread use of ARROWs.

A planar implementation technology able to straightforwardly address the third dimension is direct laser writing (DLW) [13]. This technology has recently attracted substantial attention since it has allowed to access 3D photonic band-gap materials [14], orbital-angular-momentum maintaining waveguide [15] and chip-to-chip bonding wires [16], which all are difficult or impossible to be realized by other planar waveguide technology. Using DLW, we have recently introduced a novel type of on-chip hollow core waveguide with unique properties - the light cage [17]. This structure consists of a single ring of micrometer dielectric strands arranged in a hexagonal geometry, providing light guidance in the central hollow core and owns unique properties such a diffraction-less guidance in over centimeter distances, sidewise access to the core region, extraordinary high fraction of field inside the core and compatibility with fiber circuitry.

Here we demonstrate efficient guidance of light in a singledefect dual-ring light cage over millimeter distances. The structure implemented by two-photon-polymerization (2PP) DLW is composed of two rings of high aspect-ratio polymer strands hexagonally arranged around a central hollow core and shows clear core mode formation via the PBG effect. Specifically, we 
(a)
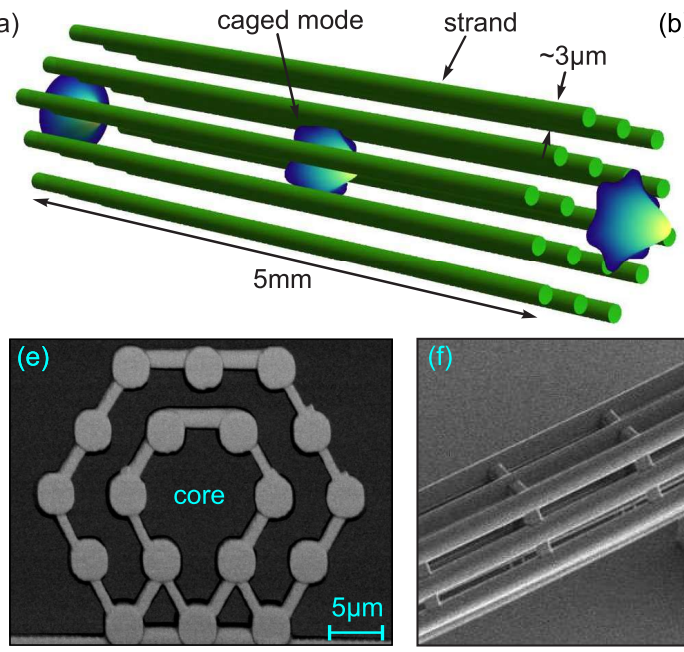

(b)

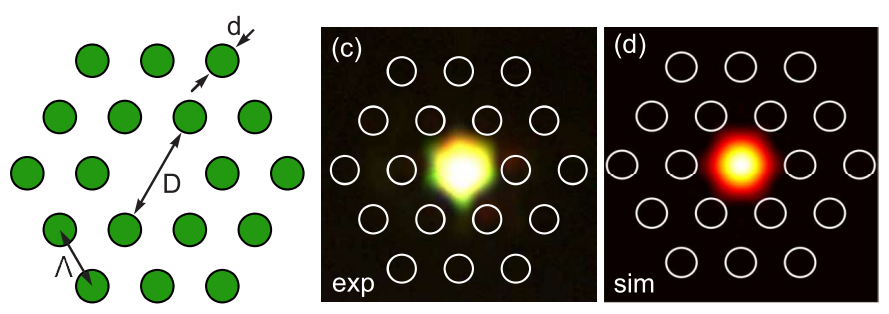

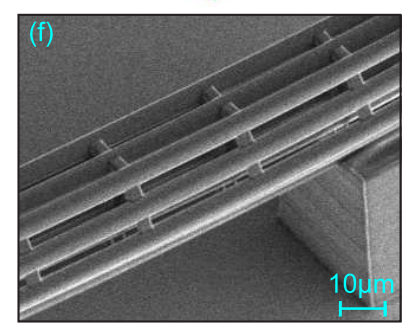
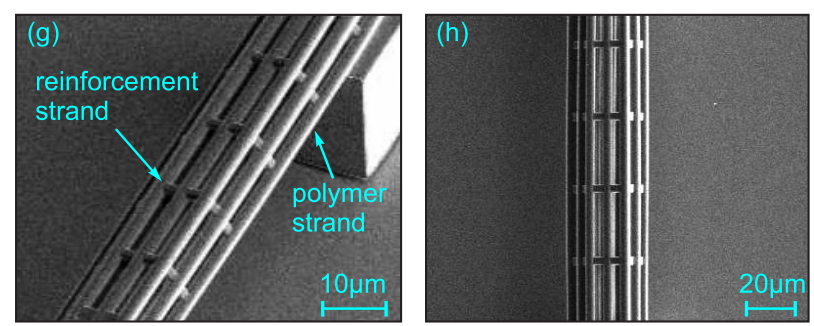

Fig. 1. The single-defect dual-ring light cage. (a) Schematic of the structure (green: dielectric strands). The colored beams refer to the light configuration at different locations in the cage (left: input beam, middle: caged mode, right: output mode). (b) Crosssection of the refractive index distribution with the relevant parameters (inter-stand distance (pitch) $\Lambda$, strand diameter $d$, core diameter $D$ ). (c) Measured mode field distribution at the output of the light cage $(d=3.6 \mu \mathrm{m}, \Lambda=7 \mu \mathrm{m})$ in case white light is launched into the core. (d) Corresponding simulated mode pattern (wavelength $894 \mathrm{~nm}$ ). (e)-(h) Various scanning electron micrographs (SEMs) images of implemented dual-ring light cages ((e): front view, (f)-(g) angled views, (h) top view).

found a series of pronounced transmission bands in the visible and near-infrared spectral domains, with simulations confirming the observations.

The cross section of the investigated structure (Figs. 1a and b) is formed by two rings of dielectric strands (strand diameter $d=$ $3.6 \mu \mathrm{m}$, center-to-center inter-strand distance (pitch) $\Lambda=7 \mu \mathrm{m}$ ) hexagonally arranged around a single-defect hollow core (core diameter $D=10.4 \mu \mathrm{m}$ ). The periodic arrangement of polymer strands leads to formation of a PBG structure, determining the optical properties of the dual-ring light cage. To reveal the modal properties, we have calculated the density of states (DOS) using finite-element modeling (Comsol Multiphyics) assuming a triangular lattice unit cell with periodic boundary conditions and constant refractive indices (simulations details can be found [18], RI strands $n_{s}=1.552$, RI background $n_{b g}=1$ )

The DOS map shown in Fig. 2 was calculated exemplarily for normalized frequencies range $58<k_{0} \Lambda<77$, corresponding to a wavelength range from $758 \mathrm{~nm}$ to $571 \mathrm{~nm}(\Lambda=7 \mu \mathrm{m})$. The map reveals several important features which allows to classify the different regions into bands (non-zero DOS, colored regions) and PBGs (zero-DOS, white regions). Inside the PBGs no photonic states are allowed in the polymer strand lattice, providing the confinement that is required for core mode formation inside the central defect region. The photonic bands can be divided into two groups. Firstly, a series of low and moderately dispersive bands are formed below the light line $\left(n_{e f f}\right.$ $\left.=n_{b g}\right)$, which strongly interact with each other and with the defect core mode. These bands correspond to mode groups with low azimuthal mode orders (e.g. $L P_{0 m}, L P_{1 m}$ ), exhibiting a large coupling coefficient with the $L P_{01}$-like hollow-core mode. As a result, energy of the core mode can tunnel through the strand lattice resonantly at the phase-matching points (equal effective indices $n_{e f f}$ ), substantially modifying its dispersion near these points and increasing its loss. Secondly, the DOS map shows a high number of highly dispersive bands across the entire investigated spectral domain, corresponding to strand modes of high radial and azimuthal mode orders (a discussion of these modes and an approximate model can be found in [19]). These modes show virtually no interaction with the core mode which is well known from revolver-type hollow-core antiresonant fibers and is due to a negligible coupling coefficient between the core mode and strand modes with a high azimuthal mode orders [8].

To investigate the impact of the number of strand rings $N_{r}$, the spectral distribution of the modal attenuation of the fundamental core mode within one selected transmission band was calculated for single, dual and triple ring light cages (Fig. 3a), revealing a significant loss reduction in case additional rings are included. To quantify this behavior, the corresponding minimal losses $\gamma_{\min }$ have been plotted vs. $N_{r}$ and fitted by the linear function $\log _{10}\left(\gamma_{\min } /(\mathrm{dB} / \mathrm{mm})\right)=a+b \cdot N_{r}$ (Fig. $3 \mathrm{~b}$, $a=1.3748, b=-2.16957)$, yielding a reduction of modal attenuation per additional ring by a factor $f_{\gamma}=\gamma\left(N_{r}+1\right) / \gamma\left(N_{r}\right)=$ $10^{b} \approx 6.8 \cdot 10^{-3}$ or $-21.7 \mathrm{~dB} /$ ring. Note that the obtained $b$-value is high compared to other types of PBG fibers which typically have small $b$-values (e.g., water core PBG fiber: $b=-0.2451$ [20], air-core PBG-fiber: $b=-0.4871$ [21]).

The presented loss value should be placed in context to characteristic sample lengths. A suitable measure is the propagation length $L_{X}$ at which the transmission reduces by a particular fraction $X$, given by $L_{X}(\lambda)=-1 / \gamma(\lambda) \cdot 10 \cdot \log _{10}(1-X)$. Here we determine the propagation length at three characteristic wavelengths $\left(\lambda_{1}=\lambda_{\text {min }}\right.$ (cyan circles in Fig. $3 c$ ), $\lambda_{2,3}=\lambda_{\min } \pm 15 \mathrm{~nm}$ (red squares, yellow triangles in Fig. 3c)) as function of $N_{r}$ for $X=5 \%$. The results indicate that a dual-ring light cage yields propagation length that are within the order of $10 \mathrm{~cm}$ (dotted horizontal line in Fig. 3c) which is larger than the typical sample lengths achievable by DLW range ( $\mathrm{mms}$ to $\mathrm{cms}$ ). Adding a third ring can in principle increase propagation lengths to hundreds of meters, while, however, these lengths are beyond to what can be achieved by DLW. Therefore, the dual-ring light cage represents the ideal compromise between fabrication effort and modal attenuation witin the context of DLW.

Note that compared to the single-defect light cages reported in our previous work [17] roughly one order of magnitude longer 




Fig. 2. Calculated density of states for a triangular lattice of dielectric rods $(\mathrm{d}=3.6 \mu \mathrm{m}, \Lambda=7.0 \mu \mathrm{m})$ in air. White regions represent PBGs (zero DOS), while colored regions refer to a finite DOS (red: low DOS, yellow: high DOS). The blue curve is the dispersion of the fundamental core mode in the central lattice defect.

propagation lengths have been achieved by the addition of the second ring $(780 \mu \mathrm{m}$ vs. $5 \mathrm{~mm}$ ) while the strong confinement provided by small core region (defined by the core area $A_{c o}$ ) is maintained in contrast to the reported twelve strand structure (twelve strand light cage $A_{c o}=339.6 \mu \mathrm{m}^{2}$, dual-ring light cage $A_{c o}=36.3 \mu \mathrm{m}^{2}$ ). Therefore the dual-ring light cage represents a promising candidate for applications that demand strong intensities maintained over millimeter distances.

The fabrication of the light cages utilizes a commercial twophoton-polymerization (TPP)-DLW system (Photonic Professional GT, Nanoscribe $\mathrm{GmbH}$ ) with a liquid negative-tone resin (IP-DIP, Nanoscribe GmbH). Based on the fabrication strategy of the single-ring light cage geometry reported in [17] multiple dual ring light cage consisting of 18 strands hexagonally surrounding a single strand defect core have been implemented on silicon chips (Figs. 1(e)-(h), $d=3.6 \mu \mathrm{m}, \lambda=7 \mu \mathrm{m})$. The length of the samples used here is $5 \mathrm{~mm}$, yielding an aspect ratio of a single dielectric strand of $\approx 1000$ overall defining a waveguide geometry with significantly higher aspect ratios compared to resonant structures $[22,23]$. The cages are raised by solid polymer blocks to a height of $35 \mu \mathrm{m}$. Structural collapsing of the cage during the drying stage has been prevented by the integration of reinforcement rings every $45 \mu \mathrm{m}$ (Fig. 1(f)-(h)). Preliminary experiments have revealed that these elements have negligible impact on the optical properties of the core mode [17].

The setup used for the optical characterization consists of a white light source, the respective sample and equipment for characterization. Light from a supercontinuum source (model NKT micro) was coupled via a 20x microscope objective into the light cage. The transmitted light after propagating through the cage was collected by another 10x objective and transferred to an optical spectrum analyzer (AQ-6315A, Ando) via a multimode fiber (FG050LGA, core size $50 \mu \mathrm{m}, \mathrm{NA}=0.22$ ). The output mode was visualized by a CCD camera and spectral narrow band filters. The setup was optimized for maximum throughput, i.e., for the situations the fundamental core mode is brightest. The transmission was normalized using by another spectrum measured without light cage.

The far-field images of the light transmitted through the dualring light cage shows a clear tightly confined core mode that resembles the six-fold symmetry of the core section (images in Fig. 4 and Fig. 1(c)). The measured spectral distribution of the transmission (Fig. 4) shows alternating regions of high and

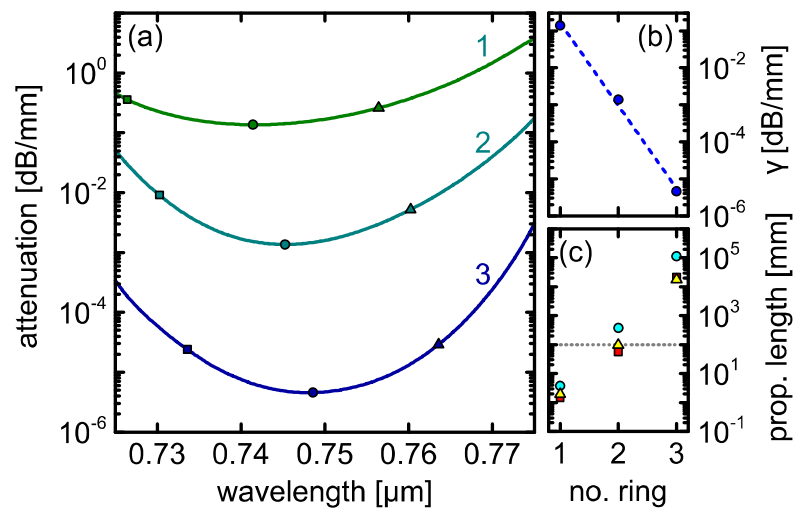

Fig. 3. (a) Simulated spectral distribution of the modal attenuation of the fundamental core mode inside light cages with different numbers of rings (indicated by the numbers) in one selected transmission band (dark green: single-ring, dark cyan: dual-ring, blue: triple-ring, $n=1.552, d=3.6 \mu \mathrm{m}$, $\Lambda=7.0 \mu \mathrm{m})$. The circles indicate the points of minimal loss $\left(\lambda_{\text {min }}, \gamma_{\text {min }}\right)$, while the squares and triangles refer to the points that are located $\pm 15 \mathrm{~nm}$ from $\lambda_{\text {min }}$. (b) Minimum modal attenuation (circles in (a)) as a function of $N_{r}$ (dashed curve: linear fit (in logarithmic scale)). (c) Propagation length as function of $N_{r}$. The circles refer to the points of minimal loss, while the squares (triangles) are related to the losses at $\lambda_{\min }-15 \mathrm{~nm}$ $\left(\lambda_{\min }+15 \mathrm{~nm}\right)$ for a transmission reduction of $X=5 \%$. The horizontal gray dotted line indicates a length of $10 \mathrm{~cm}$.

low transmission in the spectral range $450 \mathrm{~nm}<\lambda<900 \mathrm{~nm}$, which is a characteristic feature of PBG fibers that consists of high RI cylindrical strands, examples of which include hybrid chalcogenide/silica hybrid fibers [24], SF6/LLF1 fibers [25] and tellurite/silica fibers [26]. Note that both single- and dual-ring light cages show comparable spectral features despite the former being not periodic along the transverse direction. Since the photonic bands originate from coupled resonances of the polymer strands, forming supermodes (bands) and gaps between them, a distinction between anti-resonant and PBG guidance is at least in the present case questionable. The fringe contrast defined as the ratio of dip and peak transmission of one resonance shows values up to $20 \mathrm{~dB}$, reaching contrasts being comparable to those observed in $5 \mathrm{~mm}$ long hybrid $\mathrm{As}_{2} \mathrm{~S}_{3} / \mathrm{SiO}_{2}$ PBG fibers [24] emphasizing the high quality of the fabricated light cages. Note that the implemented light cage represent a hollow core waveguide with extraordinary high RI contrast $C_{R I}$ of $C_{R I}=$ $\left(n_{s}-n_{b g}\right) /\left(n_{s} \mp n_{b g}\right)=0.21\left(n_{s}=1.552, n_{b g}=1\right)$, reaching values that are close to that of the mentioned chalcogenide/silica PBG fiber $\left(C_{R I}=0.24\right)$ [24]. The transmission drop towards longer wavelengths results from a combination of the reducing reflectivity - an effect known from other kinds of hollow core fibers [27] - and from an increased impact of absorption, particular since the overlap between defect mode and strands increases. For a direct comparison between experiment and simulations, the dispersion of $n_{\text {eff }}$ of the fundamental mode (Fig. 1(d)) across the entire spectrum of experimental result including the material dispersion of the polymer has been calculated $(d=$ $3.64 \mu \mathrm{m}$, Fig. 4(a)). An analytic function of the polymer dispersion has been obtained by fitting the RI at selected wavelengths provided by Nanoscribe by a one-term Sellmeier equation, yielding $n_{p}=\left(1+A_{1} \cdot \lambda^{2} /\left(\lambda^{2}-\lambda_{1}^{2}\right)\right)^{1 / 2}$ with $A_{1}=1.3424689$ and $\lambda_{1}=0.128436 \mu \mathrm{m}$ ( $\lambda$ is given in $\left.\mu \mathrm{m}\right)$. This comparison shows 


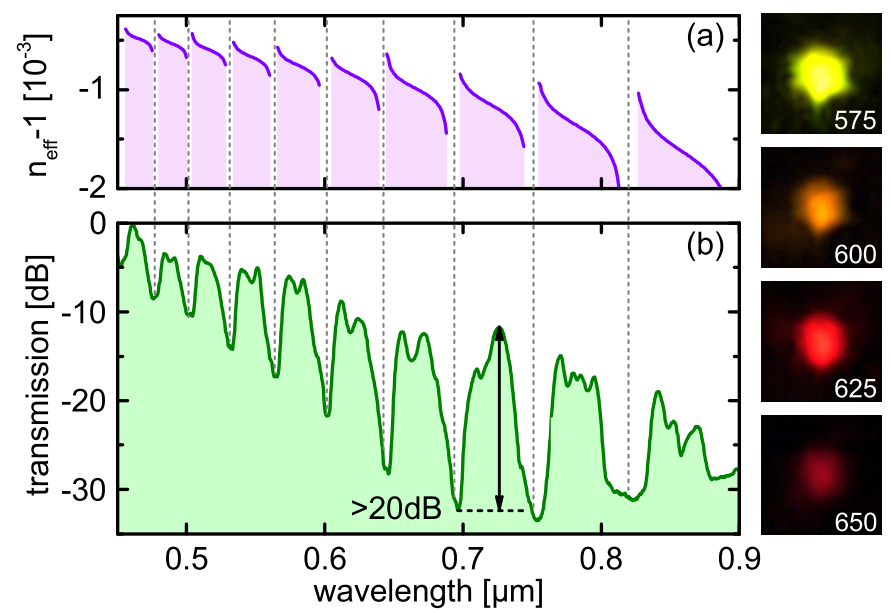

Fig. 4. (a) Spectral distribution of the real part of the effective index of the fundamental core mode (shown in Fig. 1(d)) including the material dispersion of the polymer. (b) Measured spectral distribution of the transmission of the core mode supported by the light cage shown in Fig. 1 (sample length: $5 \mathrm{~mm}$, $d=3.6 \mu \mathrm{m}, \lambda=7 \mu \mathrm{m})$. The maximum fringe contrast is $>20$ $\mathrm{dB}$. The images on the right show measured mode profiles at selected wavelengths (indicated by the numbers (in $\mathrm{nm}$ )).

that the spectral locations of the anti-crossings (i.e., avoided crossings between core mode and cladding supermodes, Fig. 4(a)) match the wavelengths of the measured transmission dips (matches indicated by the vertical dashed gray lines), revealing the excellent quality of the structure and emphasizes the degree of control on the optical properties of the light cage using DLW.

We also examined the polarization properties of the dual-ring light cage structure by measuring the transmission polarizationresolved by placing a polarizer and analyzer before and after the sample. The transmission in the crossed-polarized state is strongly suppressed by $<15 \mathrm{~dB}$, while due to lack of dynamic range of the measurement setup transmission was below noise limit in the crossed polarized state, which does not allow us to precisely state the polarization extinction ratio.

Here we demonstrated efficient guidance of light in a singledefect dual-ring light cage over millimeter distances that is integrated on a silicon chip via DLW. The structure consists of two rings of high aspect-ratio $(>1000)$ polymer strands hexagonally arranged around a central hollow core, exhibiting light guidance via the PBG effect. Experimentally we found a series of pronounced transmission bands with fringe and polarization contrasts of $>20 \mathrm{~dB}$ and $>15 \mathrm{~dB}$, respectively, within the visible and near-infrared with the core mode resembling the symmetry of the cladding. Numerical simulations confirm the experimental observations and reveal that the dual-ring arrangement represents the optimal compromise between confinement, modal attenuation and implementation effort within the light cage context.

The three dimensional nature of the light cage and in particular the side-wise access to the core region makes this hollowcore waveguide an attractive candidate for a great variety of applications, including chip-integrated gas- or liquid based spectroscopy in bioanalytics, environmental science, atomic spectroscopy or sophisticated applications in quantum technology such as magnetometry or quantum memory.

\section{FUNDING INFORMATION}

German Research Foundation via grant No. SCHM2655/8-1, Bavarian Solar Technologies Go Hybrid (SolTech) programme

\section{ACKNOWLEDGMENT}

S.A.M. additionally acknowledges the Lee-Lucas Chair in Physics. J. G. acknowledges funding from the European Commission for the Marie-Sklodowska-Curie action 797044.

\section{REFERENCES}

1. M. Nissen, B. Doherty, J. Hamperl, J. Kobelke, K. Weber, T. Henkel, and M. Schmidt, Sensors 18, 478 (2018).

2. P. S. J. Russell, P. Hölzer, W. Chang, A. Abdolvand, and J. C. Travers, Nat. Photonics 8, 278 (2014).

3. G. Epple, K. S. Kleinbach, T. G. Euser, N. Y. Joly, T. Pfau, P. S. J. Russell, and R. Löw, Nat. Commun. 5, 4132 (2014).

4. R. F. Cregan, Science. 285, 1537 (1999).

5. B. Temelkuran, S. D. Hart, G. Benoit, J. D. Joannopoulos, and Y. Fink, Nature. 420, 650 (2002).

6. M. Zeisberger, A. Tuniz, and M. A. Schmidt, Opt. Express 24, 20515 (2016).

7. Y. Y. Wang, N. V. Wheeler, F. Couny, P. J. Roberts, and F. Benabid, Opt. Lett. 36, 669 (2011).

8. B. Debord, A. Amsanpally, M. Chafer, A. Baz, M. Maurel, J. M. Blondy, E. Hugonnot, F. Scol, L. Vincetti, F. Gérôme, and F. Benabid, Optica. 4, 209 (2017).

9. G. Epple, N. Y. Joly, T. G. Euser, P. St.J. Russell, and R. Löw, Opt. Lett. 42, 3271 (2017).

10. G. Testa, Y. Huang, P. M. Sarro, L. Zeni, and R. Bernini, Opt. Lett. 35, 1584 (2010).

11. W. Yang, D. B. Conkey, B. Wu, D. Yin, A. R. Hawkins, and H. Schmidt, Nat. Photonics 1, 331 (2007).

12. D. Yin, E. J. Lunt, M. I. Rudenko, D. W. Deamer, A. R. Hawkins, and H. Schmidt, Lab on a Chip 7, 1171 (2007).

13. T. Gissibl, S. Thiele, A. Herkommer, and H. Giessen, Nat. Photonics 10, 554 (2016).

14. I. Staude, G. von Freymann, S. Essig, K. Busch, and M. Wegener, Opt. Lett. 36, 67 (2011).

15. Y. Chen, J. Gao, Z.-Q. Jiao, K. Sun, L.-F. Qiao, H. Tang, X.-F. Lin, and X.-M. Jin, Phys. Rev. Lett. 121, 233602 (2018).

16. M. R. Billah, M. Blaicher, T. Hoose, P.-I. Dietrich, P. Marin-Palomo, N. Lindenmann, A. Nesic, A. Hofmann, U. Troppenz, M. Moehrle, S. Randel, W. Freude, and C. Koos, Optica. 5, 876 (2018).

17. C. Jain, A. Braun, J. Gargiulo, B. Jang, G. Li, H. Lehmann, S. A. Maier, and M. A. Schmidt, ACS Photonics 6, 649 (2019).

18. R. Spittel, H. Bartelt, and M. A. Schmidt, Opt. Express 22, 11741 (2014).

19. R. Fatobene-Ando, A. Hartung, B. Jang, and M. A. Schmidt, Opt. Express 27, 10009 (2019).

20. G. Li, M. Zeisberger, and M. A. Schmidt, Opt. Express 25, 22467 (2017).

21. K. Saitoh and M. Koshiba, Opt. Express 11, 3100 (2003).

22. C. Sieutat, R. Peretti, J.-L. Leclercq, P. Viktorovitch, and X. Letartre, Opt. Express 21, 20015 (2013).

23. R. Artinyan, A. Benamrouche, C. Belacel, M. Kozubova, A. Berthelot, A. Jurdyc, G. Beaudin, V. Aimez, P. Rojo-Romeo, J. Leclercq, R. Peretti, X. Letartre, and S. Callard, Proc. SPIE 9127, 91270K (2014).

24. N. Granzow, P. Uebel, M. A. Schmidt, A. S. Tverjanovich, L. Wondraczek, and P. S. J. Russell, Opt. Lett. 36, 2432 (2011).

25. F. Luan, a. K. George, T. D. Hedley, G. J. Pearce, D. M. Bird, J. C. Knight, and P. S. J. Russell, Opt. Lett. 29, 2369 (2004).

26. M. A. Schmidt, N. Granzow, N. Da, M. Peng, L. Wondraczek, and P. S. Russell, Opt. Lett. 34, 1946 (2009).

27. M. Zeisberger and M. A. Schmidt, Sci. Reports 7, 11761 (2017). 


\section{FULL REFERENCES}

1. M. Nissen, B. Doherty, J. Hamperl, J. Kobelke, K. Weber, T. Henkel, and M. Schmidt, "UV Absorption Spectroscopy in Water-Filled Antiresonant Hollow Core Fibers for Pharmaceutical Detection," Sensors 18, 478 (2018).

2. P. S. J. Russell, P. Hölzer, W. Chang, A. Abdolvand, and J. C. Travers, "Hollow-core photonic crystal fibres for gas-based nonlinear optics," Nat. Photonics 8, 278 (2014).

3. G. Epple, K. S. Kleinbach, T. G. Euser, N. Y. Joly, T. Pfau, P. S. J. Russell, and R. Löw, "Rydberg atoms in hollow-core photonic crystal fibres," Nat. Commun. 5, 4132 (2014).

4. R. F. Cregan, "Single-Mode Photonic Band Gap Guidance of Light in Air," Science. 285, 1537-1539 (1999).

5. B. Temelkuran, S. D. Hart, G. Benoit, J. D. Joannopoulos, and Y. Fink, "Wavelength-scalable hollow optical fibres with large photonic bandgaps for CO(2) laser transmission," Nature. 420, 650-653 (2002).

6. M. Zeisberger, A. Tuniz, and M. A. Schmidt, "Analytic model for the complex effective index dispersion of metamaterial-cladding large-area hollow core fibers," Opt. Express 24, 20515 (2016).

7. Y. Y. Wang, N. V. Wheeler, F. Couny, P. J. Roberts, and F. Benabid, "Low loss broadband transmission in hypocycloid-core Kagome hollow-core photonic crystal fiber," Opt. Lett. 36, 669-671 (2011).

8. B. Debord, A. Amsanpally, M. Chafer, A. Baz, M. Maurel, J. M. Blondy, E. Hugonnot, F. Scol, L. Vincetti, F. Gérôme, and F. Benabid, "Ultralow transmission loss in inhibited-coupling guiding hollow fibers," Optica. 4, 209 (2017).

9. G. Epple, N. Y. Joly, T. G. Euser, P. St.J. Russell, and R. Löw, "Effect of stray fields on Rydberg states in hollow-core PCF probed by higherorder modes," Opt. Lett. 42, 3271-3274 (2017).

10. G. Testa, Y. Huang, P. M. Sarro, L. Zeni, and R. Bernini, "High-visibility optofluidic Mach-Zehnder interferometer," Opt. Lett. 35, 1584-1586 (2010).

11. W. Yang, D. B. Conkey, B. Wu, D. Yin, A. R. Hawkins, and H. Schmidt, "Atomic spectroscopy on a chip," Nat. Photonics 1, 331-335 (2007).

12. D. Yin, E. J. Lunt, M. I. Rudenko, D. W. Deamer, A. R. Hawkins, and H. Schmidt, "Planar optofluidic chip for single particle detection, manipulation, and analysis," Lab on a Chip 7, 1171-1175 (2007).

13. T. Gissibl, S. Thiele, A. Herkommer, and H. Giessen, "Two-photon direct laser writing of ultracompact multi-lens objectives," Nat. Photonics 10, 554 (2016).

14. I. Staude, G. von Freymann, S. Essig, K. Busch, and M. Wegener, "Waveguides in three-dimensional photonic-bandgap materials by direct laser writing and silicon double inversion," Opt. Lett. 36, 67-69 (2011).

15. Y. Chen, J. Gao, Z.-Q. Jiao, K. Sun, L.-F. Qiao, H. Tang, X.-F. Lin, and X.-M. Jin, "Mapping Twisted Light into and out of a Photonic Chip," Phys. Rev. Lett. 121, 233602 (2018).

16. M. R. Billah, M. Blaicher, T. Hoose, P.-I. Dietrich, P. Marin-Palomo, N. Lindenmann, A. Nesic, A. Hofmann, U. Troppenz, M. Moehrle, S. Randel, W. Freude, and C. Koos, "Hybrid integration of silicon photonics circuits and InP lasers by photonic wire bonding," Optica. 5, 876-883 (2018).

17. C. Jain, A. Braun, J. Gargiulo, B. Jang, G. Li, H. Lehmann, S. A. Maier, and M. A. Schmidt, "Hollow Core Light Cage: Trapping Light Behind Bars," ACS Photonics 6, 649-658 (2019).

18. R. Spittel, H. Bartelt, and M. A. Schmidt, "A semi-analytical model for the approximation of plasmonic bands in arrays of metal wires in photonic crystal fibers," Opt. Express 22, 11741-11753 (2014).

19. R. Fatobene-Ando, A. Hartung, B. Jang, and M. A. Schmidt, "Approximate model for analyzing band structures of single-ring hollow-core anti-resonant fibers," Opt. Express 27, 10009-10021 (2019).

20. G. Li, M. Zeisberger, and M. A. Schmidt, "Guiding light in a water core all-solid cladding photonic band gap fiber - an innovative platform for fiber-based optofluidics," Opt. Express 25, 22467 (2017).

21. K. Saitoh and M. Koshiba, "Leakage loss and group velocity dispersion in air-core photonic bandgap fibers," Opt. Express 11, 3100-3109 (2003).

22. C. Sieutat, R. Peretti, J.-L. Leclercq, P. Viktorovitch, and X. Letartre, "Strong confinement of light in low index materials: the photon cage,"
Opt. Express 21, 20015-20022 (2013).

23. R. Artinyan, A. Benamrouche, C. Belacel, M. Kozubova, A. Berthelot, A. Jurdyc, G. Beaudin, V. Aimez, P. Rojo-Romeo, J. Leclercq, R. Peretti, $X$. Letartre, and S. Callard, "Optical characterization of active photon cages," Proc. SPIE 9127, $91270 \mathrm{~K}$ (2014).

24. N. Granzow, P. Uebel, M. A. Schmidt, A. S. Tverjanovich, L. Wondraczek, and P. S. J. Russell, "Bandgap guidance in hybrid chalcogenide-silica photonic crystal fibers," Opt. Lett. 36, 2432 (2011).

25. F. Luan, a. K. George, T. D. Hedley, G. J. Pearce, D. M. Bird, J. C. Knight, and P. S. J. Russell, "All-solid photonic bandgap fiber," Opt. Lett. 29, 2369-2371 (2004).

26. M. A. Schmidt, N. Granzow, N. Da, M. Peng, L. Wondraczek, and P. S. Russell, "All-solid bandgap guiding in tellurite-filled silica photonic crystal fibers," Opt. Lett. 34, 1946-1948 (2009).

27. M. Zeisberger and M. A. Schmidt, "Analytic model for the complex effective index of the leaky modes of tube-type anti-resonant hollow core fibers," Sci. Reports 7, 11761 (2017). 